\title{
Inside the Survivor's Mind: The Portrayal of the content of rumination of breast cancer survivors experiencing post-traumatic growth
}

\author{
Putri Ayu Widyautami* \\ *Faculty of Psychology, Mercu Buana University, Jakarta, Indonesia \\ *E-mail: putriayu.widyautami@gmail.com
}

\begin{abstract}
The objective of this study is to describe the substance of the rumination of breast cancer survivors experiencing post-traumatic growth during the stages of pre-diagnosis, living with cancer, living through cancer and living beyond cancer. The research used a qualitative approach with a phenomenological approach. Participants selected to be interviewed was included based on the score of the short form of the post-traumatic growth inventory. Subjects were three breast cancer survivors who have experienced post-traumatic growth. The results showed that the content of the rumination of breast cancer survivors experiencing post-traumatic growth comprises ten main themes: death, family, work, finances, social interaction, health, appearance, cancer as a positive experience, religion/spirituality, and life. However, there are also a number of sub-themes for each of the ten themes, which are different for each subject and each phase. Practitioners and caregivers can use the results of this study to help patients deal with their ruminations and achieve post-traumatic growth.
\end{abstract}

Keywords: breast cancer survivor; post-traumatic growth; rumination

\section{Introduction}

Cancer is a disease which caused the second highest number of deaths in the world, after heart disease (Ministry of Health for the Republic of Indonesia, 2014). In 2008, approximately 7.6 million people, or $13 \%$ of the world's population, lost their lives to cancer (World Health Organization, 2015). Breast cancer is the most common type of cancer in Indonesia, with a prevalence of $18.6 \%$ (National Cancer Control Committee, 2015). According to Park (2013), a person was said to be a breast cancer survivor after being diagnosed with breast cancer and continues to be declared a breast cancer survivor throughout the period of treatment and for the rest of her life.

Breast cancer is a chronic illness that can threaten the life of the patient. The experiences of individuals suffering from life-threatening events are presented in edition 5 of the Diagnostic and Statistical Manual of Mental Disorders, and such events are said to have the potential to produce trauma (American Psychiatric Association, 2013).
A traumatic event can cause psychological stress and usually produces adverse effects on the individual. However, recent research studies have shown that breast cancer, which can be classified as a traumatic event, has the potential to trigger positive personal changes, and this is referred to as post-traumatic growth (PTG). Some studies report that $50 \%$ to $90 \%$ of breast cancer survivors have been proven to experience post-traumatic growth (Joseph \& Linley, 2008).

Model of post-traumatic growth consists of seven key elements, i.e characteristics of the individual, characteristics of the traumatic events, distress management, sociocultural influences, life narrative developments, rumination, and wisdom. However, between these seven elements, the relationship between rumination and post-traumatic growth is one area that has the potential to be explored for further research compared with the other six elements (Calhoun \& Tedeschi, 2006).

Calhoun \& Tedeschi (2006) define rumination as the involvement of a cognitive process in the understanding of the trauma and the consequences there of rumination in breast cancer patients begins 
in the pre-diagnosis phase (Sonja Lyubomirsky, 2006). Furthermore, Park (2013) states that in general, the content of the rumination of cancer survivors may differ according to the three stages through which a survivor passes, in the phase of the living with cancer, living through cancer, and living beyond cancer stages. The content of the rumination of the individual experiencing trauma usually includes unpleasant elements related to the traumatic events, and it is likely that most of the individual's thoughts are related to the traumatic events. Nevertheless, repetitive thoughts about other types of content may also occur after a traumatic event, and further studies may cover these different types of content (Calhoun \& Tedeschi, 2006).

Based on the search results, the writer discovered two previous research studies on the topic of rumination. One study by Zhang Lei (2012), involving 21 cancer patients. This study reports that the content of the rumination of the research subjects deliberately focused on themes of (1) how to deal with the illness they were suffering from; (2) how to accept the illness and appreciate it; (3) the process of gradual change that they experienced, from resisting to eventually accepting the illness, and; (3) themes of how they focused on balance and harmony in their bodies and minds. Another research study by Edwards, Tang, Wright, Salkovskis, \& Timberlake (2011), investigating 20 chronically ill patients, reports that the content of the rumination of the subjects included themes related to the pain they were experiencing as a result of the illness, their jobs, families, relationships with other people, finances, and overall health. However, researcher still found no previous studies about the content of rumination of subjects experiencing posttraumatic growth.

The fact that there has been no previous research studies found the connection between rumination and post-traumatic growth opened up the possibility for further study in this area. There were some anticipation that the results of the present study will give a description of the content of the rumination of breast cancer survivors experiencing posttraumatic growth. The dynamics of the changes in the content of the rumination from the pre-diagnosis phase to the of living with cancer, living through cancer, and living beyond cancer stages will provide important information for practitioners and caregivers in helping cancer survivors deal with their rumination and achieve post-traumatic growth. In addition, it is expected that this study will offer a new perspective for other researchers about the content of rumination and post-traumatic growth.

\section{Methods}

This research used a qualitative approach with a phenomenological approach. Phenomenology, which endeavors to disclose, analyze, and understand a phenomenon and its specific and unique context as experienced by an individual, up to the level of "conviction" of the individual concerned (Herdiansyah, 2015). In this present study, additional quantitative data is included as supporting data. Quantitative data is used as an indicator for identifying the research subjects.

Instrument and measurement. The quantitative data was collected through a short form of the posttraumatic growth inventory or PTG-SF. PTGI-SF had very high internal reliability and an adjusted correlation with the PTGI of over .90 across a variety of samples. The 10-item of PTGI-SF measured positive changes following adversity across five PTG dimensions: relating to others, new possibilities, personal strength, spiritual change and appreciation of life. Each item was rated along a 6point Likert-type scale $(0=$ 'I did not experience this change as a result of my illness' to $5=$ 'I experienced this change to a very great degree as a result of my illness'). The scale was scored according to the five subscales, with higher scores demonstrating a greater level of each particular dimension of PTG (Can, et al, 2010). Meanwhile, the qualitative data was collected through semistructured interviews and observation.

Sample. The sampling technique used in this research is purposive sampling. The criteria for the research subjects were: (a) Female; (b) At least 18 years of age; (c) Diagnosed with breast cancer for more than 1 month; (d) Having an experience of post-traumatic growth (identified based on the score obtained from a standard of measurement known as a short form of the post-traumatic growth inventory).

Procedure. The writer held brief interviews with 10 breast cancer patients at Gatot Soebroto Army Hospital and asked them to complete the questions on the short form post-traumatic growth inventory (PTGI-SF). Of these ten patients, the three patients with the highest scores were then chosen to be used as the research subjects. These three patients are identified with the initials $\mathrm{W}, \mathrm{Y}$, and S. Subject W was 42 years old and had been diagnosed with cancer for three years and ten months. Subject Y was 65 years old and had been diagnosed with cancer for eleven years and three months. Subject S was 64 years old and had been diagnosed with cancer for eighteen years and ten months. 
Table 1.

Total Score of PTGI-SF scale from 10 breast cancer patients

\begin{tabular}{cc}
\hline Initial & Total score \\
\hline R & 20 \\
S & 29 \\
SA & 35 \\
D & 36 \\
R & 32 \\
A & 43 \\
C & 31 \\
W & 44 \\
Y & 45 \\
S & 49 \\
\hline
\end{tabular}

The semi-structured interviews for 3 subjects began with the following explanation about rumination: "Rumination means thinking about something repeatedly, or over and over again. Rumination may happen either intentionally or unintentionally". The subjects were then asked questions based on the time periods: pre-diagnosis, living with cancer, living through cancer, and living beyond cancer. The prediagnosis phase refers to the period of time when the subject first becomes aware of the symptoms of breast cancer. The phase living with cancer refers to the period when the diagnosis is made and active treatment is given. The phase living through cancer begins after the active treatment has finished. The phase living beyond cancer begins five years after the living through cancer phase, or when the potential of cancer recurring or metastasizing is very small. For each phase, the subjects were asked the following questions: (1) what did you frequently ruminate about during this time? ; (2) How often did you ruminate about this theme? ; (3) Did you experience any emotional or other changes during or following the rumination about this theme? ; (4) What caused you to stop ruminating about this theme?
The data analysis in this research study involves the three stages of data analysis from the phenomenological research model, namely, the preliminary stage, the horizontalization stage, and the cluster of the meaning stage (Creswell, 2007). First, in the preliminary stage, the researcher transcribed in verbatim the entire recording of the results of the interviews. Second, in the horizontalization stage, the researcher marked the important statements that were relevant to the topics contained in the verbatim interviews. And third, in the cluster of the meaning stage, the researcher classified the important statements already marked into different themes. A triangulation perspective was used in this research by interviewing family members of the research subjects who acted as informants for the research study.

\section{Results}

Subjects $\mathrm{Y}$ and $\mathrm{S}$ were in the living beyond cancer phase, while subject $\mathrm{W}$ was in the living through cancer phase. In the case of subject $\mathrm{W}$, rumination began during the living with cancer phase. The reason for this was that the subject was not aware of any symptoms of breast cancer in her body prior to undergoing a medical examination. In the case of subjects $\mathrm{Y}$ and $\mathrm{S}$, rumination began during the prediagnosis phase, or when the subjects became aware that their bodies were showing symptoms of breast cancer. The result of the rumination during the prediagnosis phase influenced $\mathrm{Y}$ and $\mathrm{S}$ in such a way as to postpone undergoing a medical examination. This finding supports the findings of previous research studies which report that breast cancer patients begin to engage in rumination from the time they are first aware of their symptoms and patients who engage in extended rumination are more inclined to postpone medical examination (Sonja Lyubomirsky, 2006).

Based on the results of the analysis, it was found that in this research study there were ten themes that filled the rumination of the breast cancer survivors were experiencing post-traumatic growth. These themes are death, family, work, finances, social interaction, health, appearance, and cancer as a positive experience, religion/spirituality, and life. 
Table 2.

Themes and Sub-category Themes that filled the rumination of breast cancer survivor were experiencing post-traumatic growth

\begin{tabular}{|c|c|}
\hline Theme & Sub-category theme \\
\hline Death & - The threat of death \\
\hline Family & $\begin{array}{l}\text { - Nuclear family } \\
\text { •Extended family }\end{array}$ \\
\hline Work & $\begin{array}{l}\bullet \text { Closed business } \\
\bullet \text { Stopped working }\end{array}$ \\
\hline Finances & $\begin{array}{l}\text { - Source of medical expenses } \\
\text { for breast cancer }\end{array}$ \\
\hline Social Interaction & $\begin{array}{l}\text { - Social support } \\
\text { - Other breast cancer survivors }\end{array}$ \\
\hline Health & $\begin{array}{l}\text { - Treatment process for breast } \\
\text { cancer } \\
\text { - The potential of breast cancer } \\
\text { recurrence } \\
\text { - Healthy lifestyle }\end{array}$ \\
\hline Appearance & $\begin{array}{l}\text { - Change in appearance } \\
\text { - Manipulation of appearance }\end{array}$ \\
\hline $\begin{array}{l}\text { Breast cancer as a } \\
\text { positive experience }\end{array}$ & $\begin{array}{l}\text { - Breast cancer is a challenge } \\
\text { - Breast cancer treatment } \\
\text { process as a positive } \\
\text { experience } \\
\text { - Positive changes as a result of } \\
\text { breast cancer }\end{array}$ \\
\hline $\mathrm{R} / \mathrm{S}$ themes & $\begin{array}{l}\bullet \mathrm{R} / \mathrm{S} \text { practice } \\
\bullet \mathrm{R} / \mathrm{S} \text { beliefs } \\
\bullet \mathrm{R} / \mathrm{S} \text { transformation. }\end{array}$ \\
\hline Life & $\begin{array}{l}\bullet \text { Meaning of life } \\
- \text { Life goals }\end{array}$ \\
\hline
\end{tabular}

\section{A. Death}

Rumination about the threat of death due to breast cancer was experienced by all three of the subjects during the living with cancer phase. The frequency of rumination on this theme was quite high in the early stages of diagnosis. The adverse effect of this type of rumination was that it made the subject feels sad and angry. The reason why the rumination ceased was a change in life priorities. Subject $\mathrm{Y}$ and $\mathrm{S}$ decided to give more priority to religious devotion. Meanwhile, Subject W decided to prioritize more of her time for charity work. According to the goal progress theory, individuals who believe that their death is imminent will reevaluate their goal values and their ability to chase these goals. This evaluation encourages a change in priorities. (Martin \& Tesser, 2006).

\section{B. Family}

All three subjects' experience rumination about the family during the living with cancer and living through cancer stages. This theme included ruminating about the subject's close family members, or nuclear family, as well as their extended family. The frequency of this type of rumination was quite high during the early stages of diagnosis and the treatment process. This type of rumination produced both adverse and positive effects. In the case of subject $\mathrm{W}$, rumination about her close family made the subject reluctant to communicate her thoughts and feelings about the breast cancer to her family members because she was afraid it will make them feel burdened. This finding is concurrent with the views of Calhoun, Cann, and Tedeschi (2010), who state that an individual coming from a collective culture is interdependent and inclined to find it easier to express positive emotions rather than adverse emotions in purpose to maintain the harmony of the group. On the other hand, the positive effect felt by subject $\mathrm{W}$ was increased motivation to become well. Subjects S and Y from their ruminations about their close family also felt this positive effect. Meanwhile, the adverse effect experienced by subjects $\mathrm{S}$ and $\mathrm{Y}$ was a feeling of disappointment when they ruminated on the lack of emotional support from their extended families.

\section{Work}

This theme filled the subjects' rumination during the living with cancer phase. The adverse effect of this type of rumination was that it made the subjects anxious. The frequency of this rumination was quite high during the early stages of diagnosis and the treatment process. In the case of subject $Y$, rumination about work was what caused her the greatest burden at that time and prevented her from focusing on her health and the treatment process she had to undertake. Based on the goal progress theory, information related to unmet goals automatically seizes the attention of the subject even though the subject is consciously focusing on other matters (Martin \& Tesser, 2006). A rumination on this theme ceased after the subjects found a solution to their problems. Subject W and $\mathrm{S}$ decided to close their businesses, while subject Y was given special dispensation by her company, allowing her to work fewer hours. 


\section{Finances}

This theme was present in the rumination of subject $\mathrm{W}$ and $\mathrm{Y}$ during the living with cancer phase. The frequency of this type of rumination was very high during the treatment process. The adverse effect of ruminating about finances was that it caused the subjects to become anxious. In the case of subject $S$, the rumination on the theme of finances appeared during the pre-diagnosis phase and continued during the living through cancer phase. According to subject $S$, rumination about finances was the thing that caused her the greatest burden during that time. Ruminating about the high cost of breast cancer treatment led subject $\mathrm{S}$ to postpone undergoing a medical examination for one year after she first became aware of the symptoms of breast cancer in her body. During that year, subject $\mathrm{S}$ experienced alternative treatment because she thought it would cost less. In the case of subject $S$, rumination about finances repeatedly occurred during the living with cancer and living through cancer stages. This was because that subject $\mathrm{S}$ never had the confidence to implement the solution that she believed would resolve her problem, which was to use the money from selling her house to pay for the cost of treatment. Based on the perspective of the response style theory, even though an individual manages to find a solution to her problems, her ruminations may cause her to lack confidence and prevent her from implementing the solution (Nolen-Hoeksema, Wisco, \& Lyubomirsky, 2008).

\section{E. Health}

The theme of health includes rumination about the breast cancer treatment process, the potential of recurrence, and the subject's desire to implement a healthy lifestyle. In the case of subject $\mathrm{W}$ and $\mathrm{S}$, the rumination on the sub-theme of cancer treatment appeared during the living with cancer phase. In subject $\mathrm{Y}$, this sub-theme had already been present in her rumination since the prediagnosis phase. Rumination about the adverse effects of breast cancer treatment made subject $\mathrm{Y}$ afraid and led her to postpone further medical examination for two months after first of her awareness of the symptoms of breast cancer. The sub-themes related to the potential of recurrence and healthy lifestyle was present in the rumination of subject $\mathrm{W}, \mathrm{Y}$, and $\mathrm{S}$ during the living through cancer phase. This finding is concurrent with the views of Park (2013), who states that during the living with cancer phase, the most intensive and immediate type of rumination is likely to be focused on medical issues. Meanwhile, during the living through cancer phase, rumination tends to be more focused on the potential of recurrence of the disease. The adverse effect of rumination on the treatment process and potential of recurrence was that it led to anxiety in the subjects. Rumination about healthy lifestyle, meanwhile, affected lowering the subjects' anxiety levels.

\section{F. Social interaction}

The theme of social interaction includes rumination about social support and other breast cancer survivors. This type of rumination appeared during the living with cancer phase. In the case of subject $\mathrm{Y}$ and $\mathrm{S}$, the rumination on this theme continued up to the living beyond cancer phase, while in the case of subject $\mathrm{W}$, this type of rumination continued until the living through cancer phase. The positive effect of this rumination was to make the subject feel content with their social connections and have more appreciation for the emotional support given to them by others. This is in line with the opinion of Joseph and Linley (2008) who state that the condition of a cancer patient, who requires a higher level of physical care and emotional support from other people than prior to the illness, may produce interpersonal experiences that encourage the cancer patient to re-evaluate their relationships with other people. Furthermore, cancer patients' condition may increase their appreciation for other people and make them feel content in certain relationships.

\section{G. Appearance}

A rumination on the theme of appearance was caused by the physical changes that the three subjects experienced as a result of the mastectomies and chemotherapy they underwent. In the case of subjects $\mathrm{Y}$ and $\mathrm{S}$, rumination about their appearance only occurred during the living with cancer phase and ended when decided manipulate their physical appearance by using a special kind of bra and a wig. In the case of subject $\mathrm{W}$, however, the rumination on this theme began during the living with cancer phase and was repeated during the living through cancer phase. Subject $\mathrm{W}$ admitted that her rumination about appearance caused her the greatest burden during these stages. The adverse effect of rumination about appearance was that it caused subject $\mathrm{W}$ to feel ashamed and develop low self-esteem. This type of rumination also led subject $\mathrm{W}$ to postpone the breast cancer treatment process for two weeks. Subject W ceased to ruminate about her appearance after joining CISC and making friends 
with other breast cancer survivors. The selfconfidence shown by other breast cancer survivors encouraged subject $\mathrm{W}$ to stop ruminating about her appearance and to feel more confident. She thought that if other cancer survivors felt confident using a special bra and a wig to enhance their appearance, then she would also do the same. This is concurrent with the findings of Calhoun, Cann, \& Tedeschi (2010), who state that the ruminations of interdependent individuals about traumatic events will be filtered based on how these events are viewed by other people in their reference group.

\section{H. Breast cancer as a positive experience}

This theme describes the content of the subjects' rumination about the positive things that they associated with breast cancer. In this type of rumination, the subjects viewed the cancer treatment process as an enjoyable activity because it made them go out and meet other breast cancer survivors at the hospital. Also, the subjects viewed breast cancer as a challenge and saw that their experience dealing with breast cancer had produced many positive changes. This type of rumination was experienced by all of the subjects repeatedly during the living with cancer and living through cancer stages and accelerated their process of adaptation to the stressful conditions caused by the breast cancer treatment process. According to Calhoun, Cann, \& Tedeschi (2010), individuals from Eastern cultures are inclined to seek ways to adapt to conditions of stress that they encounter.

\section{Religion/spirituality}

The presence of religious/spiritual themes in the rumination of the subjects supports the statement of Joseph and Linley (2008), namely that many cancer patients choose to ruminate about their belief (or non-belief) in God and spirituality. The religious/spiritual sub-themes found in this research include religious/spiritual practice, religious/spiritual beliefs, and religious/spiritual transformation. The sub-theme religious/spiritual practice describes the rumination of the subjects, which involved an increased frequency and intensity in their religious devotion. The subtheme religious/spiritual beliefs refer to the subjects' rumination about their acceptance of cancer as God's will and the belief that God would help them to face their destiny. The sub-theme religious/spiritual transformation describes the subjects' rumination about their desire to deepen their knowledge about religion. The subjects' rumination on religious/spiritual themes encouraged them to feel thankful to God and reduced the anxiety they experienced when dealing with their illness. In the case of subject $\mathrm{Y}$ and $\mathrm{S}$, religious/spiritual themes appeared during the living with cancer phase and continued into the living beyond cancer phase. In subject W, meanwhile, this theme appeared during the living with cancer phase and the living through cancer phase. In addition, in the case of subject $\mathrm{W}$, there was a difference in the sub-theme of religious /spiritual beliefs. During the living with cancer phase, the subject often ruminated about the reason why God had given her breast cancer. This made the subject feel angry and disappointed. The subject ceased this type of rumination when she became aware that it was wrong for her always to be overthinking about a problem over which she had no control and for not believing that God would help her to overcome the problem.

\section{J. Life}

The theme of life includes rumination about the meaning and goals of life. This type of rumination appeared during the living through cancer phase. In the case of subjects' $\mathrm{Y}$ and $\mathrm{S}$, this type of rumination continued up to the living beyond cancer phase. This is in line with the findings of Joseph and Linley (2008), who state that the possibility of dying and changes in the patient's routine caused by cancer may encourage the patient to ruminate about themes of life. It includes, how to understand the meaning of life, life values, how to appreciate life, life goals, and other ways of approaching life. All three subjects viewed life as a valuable opportunity that they felt they should fight. The life goals that the subjects wished to achieve were: (1) helping other breast cancer survivors to overcome their problems related to breast cancer; and (2) developing a closer relationship with God.

\section{Discussion \& Conclusion}

Discussion. Some of the limitations of this research need to be addressed to improve in further research are:

1. This research investigated the themes that become the content of the rumination of breast cancer survivors experiencing post-traumatic growth. However, the study didn't explain the effects of these rumination themes on posttraumatic growth. Therefore, further research is required to investigate more deeply the relationship between the themes of rumination and post-traumatic growth. 
2. This research described the rumination of the subjects during the stages of pre-diagnosis, living with cancer, living through cancer, and living beyond cancer. However, further studies on research subjects in the same stages need to be carried out to generalize the research results of a more homogeneous group of subjects.

3. The current study outlines the positive and adverse effects of certain themes of rumination on the research subjects. However, in this research, it was discovered that some types of rumination content did not have the same effects on all three of the research subjects. Further research is required to investigate the differences in the effects of different types of rumination content on cancer patients.

4. In this research study, the rumination of subjects $\mathrm{Y}$ and $\mathrm{S}$ began during the prediagnosis phase or prior to the living with cancer phase. The themes of subject Y's rumination (health) and subject $S$ 's rumination (finances) caused the subjects to postpone undertaking a medical examination after becoming aware of the symptoms of breast cancer in their bodies. Further research may explore in more depth the relationship between the themes of rumination and the decision of the subjects to postpone medical examinations.

5. The results of the present study provide a general picture about the content of the subjects' thoughts which replaced or ended their rumination, including: (A) thoughts about manipulating their appearance ended their rumination about changes in appearance due to undergoing mastectomies and chemotherapy; (B) thoughts about changing their priorities in life ended their rumination about death; and (C) thoughts about a healthy lifestyle ended their rumination about the potential of recurrence of the cancer. The researcher believes this content is important to be studied further. Through further research, we expect to discover more about this content and can be used by health practitioners (nurses, doctors, and psychologists) to be able to help breast cancer patients to prevent and stop their rumination with adverse themes.

6. This study has successfully described the themes become the content of the rumination of the subjects during the pre-diagnosis, living with cancer, living through cancer, and living beyond cancer stages. However, the data collection that took place over a single period of time (January-March 2016) may create a bias caused by the research subjects' memories. Therefore, further studies may longitudinally investigate the content of rumination in order to eliminate this bias and to learn in greater depth about the dynamics of change in the content of the subjects' rumination in each period.

7. The current research has succeeded in exploring a certain amount of the effect of a collective culture and Eastern culture on the content of the rumination of the research subjects. Further research is needed to develop a cross-cultural study, which will discover more about the different effects of various kinds of culture on the content of rumination.

Conclusion. The content of the rumination of breast cancer survivors experiencing posttraumatic growth consists of 10 themes, namely death, family, work, finances, social interaction, health, appearance, cancer as a positive experience, religion/spirituality, and life. However, there are also a number of sub-themes for each of the 10 themes, which are different for each subject and during each phase.

\section{References}

American Psychiatric Association. (2013). Diagnostic and statistical manual of mentar disorders fifth edition. Arlington: American Psychiatric Association.

Calhoun, L. G., \& Tedeschi, R. (2006). Handbook of posttraumatic growth. New York: Psychology Press.

Calhoun, L. G., Cann, A., \& Tedeschi, R. G. (2010). The Posttraumatic Growth Model: Sociocultural. In $\mathrm{T}$. Weiss, \& R. Berger, Posttraumatic Growth and Culturally Competent Practice (pp. 8-10). New Jersey: John Wiley \& Sons.

Cressweell, J. W. (2007). Qualitative inquiry and research design: Choosing among five approaches. California: Sage Publication.

Edwards, M. J., Tang, N. K., Wright, A. M., Salkovskis, P. M., \& Timberlake, C. M. (2011). Thinking about thinking about pain: A qualitative investigation of rumination in chronic pain. Pain Manage, 1-13.

Herdiansyah, H. (2015). Metodologi penelitian kualitatif untuk ilmu psikologi. Jakarta: Salemba Empat.

Joseph, S., \& Linley, A. P. (2008). Trauma, recovery, and growth. New Jersey: John Wiley \& Sons.

Kementrian Kesehatan Republik Indonesia. (2015). Infodatin Kanker. Jakarta: Pusat Data dan Informasi Kementerian Kesehatan RI.

Komite Nasional Penanggulangan Kanker. (2015). Panduan nasional penanganan kanker payudara. Jakarta: Kementerian Kesehatan Republik Indonesia.

L. Park, C. (2013). Positive Psychology Perspectives Across the cancer continuum: Meaning spirituality and growth. In B. I.Carr, Psychological aspects of cancer (pp. 101102). New York: Springer. 
Martin, L. L., \& Tesser, A. (1996). Some ruminative thought. In J. Robert S.Wyer, Ruminative thoughts: Advances in social cognition Volume 9 (pp. 1-14). New Jersey: Lawrence Erlbaum Associates.

Martin, L. L., \& Tesser, A. (2006). Extending the goal progress theory of rumination: Goal reevaluation and Growth. In L. J. Sanna, \& E. C. Chang, judgment over time (pp. 145-162). New York: University Press.

Nolen-Hoeksema, S., Wisco, B. E., \& Lyubomirsky, S. (2008). Rethinking Rumination. Association for Psychological Science, 3 (5), 400-403. doi: 10.1111/j.1745-6924.2008.00088.x

Sonja Lyubomirsky, F. K. (2006). Ruminative response styles and delay of seeking diagnosis and delay of seeking diagnosis. Journal of Social and Clinical Psychology, 25(3), 276-304.

World Health Organization. (2015). Cancer. Retrieved from World Health Organization website: http://www.who.int/mediacentre/factsheets/fs297/en

ZhangLei, \& FanHongXia. (2012). Qualitative Study on Content of Cancer Patients' Deliberate Rumination. Thesis. Retrieved from http://www.topresearch.org/showinfo-229-883793-

$0 . h$ tml 